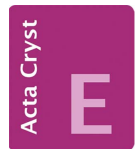

CRYSTALLOGRAPHIC COMMUNICATIONS

ISSN 2056-9890

Received 1 September 2016

Accepted 18 September 2016

Edited by G. Smith, Queensland University of Technology, Australia

Keywords: crystal structure; silver(I) complex; non-steroidal anti-inflammatory drug; diclofenac; two-dimensional coordination polymer.

CCDC reference: 1500646

Supporting information: this article has supporting information at journals.iucr.org/e

\section{Crystal structure of a mixed-ligand silver(I) complex of the non-steroidal anti-inflammatory drug diclofenac and pyrimidine}

\author{
Sevim Hamamci Alisir ${ }^{a *}$ and Necmi Dege ${ }^{b}$
}

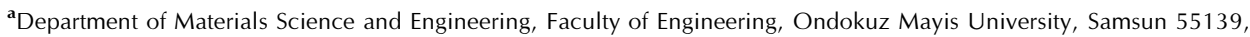
Turkey, and ${ }^{\mathbf{b}}$ Department of Physics, Faculty of Arts and Sciences, Ondokuz Mayis University, Samsun 55139, Turkey. *Correspondence e-mail: sevimh@omu.edu.tr

In the title mixed-ligand silver(I) coordination polymeric complex with the nonsteroidal anti-inflammatory drug diclofenac $\left(\mathrm{C}_{14} \mathrm{H}_{11} \mathrm{Cl}_{2} \mathrm{NO}_{2}\right)$ (diclH) and pyrimidine (pym), namely poly[ $\left\{\mu_{2}-2-[2-(2,6-d i c h l o r o a n i l i n o) p h e n y l]\right.$ acetato$\left.\kappa^{2} O: O^{\prime}\right\}\left(\mu_{2}\right.$-pyrimidine- $\left.\kappa^{2} N^{1}: N^{3}\right)$ silver(I)], $\quad\left[\mathrm{Ag}\left(\mathrm{C}_{14} \mathrm{H}_{10} \mathrm{Cl}_{2} \mathrm{NO}_{2}\right)\left(\mathrm{C}_{4} \mathrm{H}_{4} \mathrm{~N}_{2}\right)\right]_{n} \quad$ or $[\mathrm{Ag}(\mu \text {-dicl })(\mu \text {-pym })]_{n}$, the very distorted tetrahedral $\mathrm{AgN}_{2} \mathrm{O}_{2}$ coordination centres comprise two $\mathrm{N}$-atom donors from bridging pym ligands $[\mathrm{Ag}-\mathrm{N}=$ 2.381 (3) and 2.412 (3) $\AA$ ] and two carboxylate O-atom donors from dicl ligands $[\mathrm{Ag}-\mathrm{O}=2.279(2)$ and $2.280(2) \AA]$, which bridge $\mathrm{Ag}$ atoms, giving a centrosymmetric dinuclear units with a short Ag...Ag separation $[2.8931(5) \AA]$. Within the units are short intraligand $\mathrm{C}-\mathrm{Cl} \cdots \pi(\mathrm{pym})$ interactions [3.6409 (15) $\AA$ ]. The units are linked through the bridging $\mathrm{N}$ atoms of the pym ligand into a two-dimensional sheet-polymer structure lying parallel to (100) and stabilized by inter-ring $\pi-\pi$ interactions between the pym ligands $[C g \cdots C g=3.4199(17) \AA]$. Additional inter-unit $\mathrm{C}-\mathrm{H} \cdots \mathrm{O}$ and $\mathrm{C}-\mathrm{H} \cdots C g$ hydrogen-bonding interactions between the sheets give an overall threedimensional structure.

\section{Chemical context}

The design of coordination polymers based on silver(I) has been studied extensively in recent years because of their various structural topologies as well as photoluminescent properties and antimicrobial activity. These studies have shown that short $\mathrm{Ag} \cdots \mathrm{Ag}$ separations are one of the most important factors for the manifestation of such properties [Yam \& Lo, 1999; Pyykkö et al., 1997; Wang \& Cohen, 2009; Zhang et al., 2009, Njogu et al., 2015; Nomiya et al., 2000]. On the other hand, it is known that to construct extended coordination networks with polynuclear metal-based structures, ligands of various binding sites and shapes have to be taken into account. At this stage, confidence in accomplishing this goal is based upon the sophisticated selection and utilization of suitable multifunctional organic ligands with certain features, such as being a multiple donor and having versatile bonding modes or the ability to take part in hydrogen bonding. Aromatic carboxylate derivatives have therefore been of interest in coordination and supramolecular chemistry.

The chemical classes of non-steroidal anti-inflammatory drugs (NSAIDs) consist of salicylate derivatives, phenylalkanoic acids, oxicams, anthranilic acids, sulfonamides and furanones (Weder et al., 2002). These compounds are some of the most commonly used medications to reduce pain, and 
diclofenac (dicl), [2-(2,6-dicholoroanilino)phenylacetic acid], is a member of the group of phenylalkanoic acids. Additionally, NSAIDs are used as anti-inflammatories, antipyretics and antitumor drugs. (Kim et al., 2004; Ribeiro et al., 2008; Duffy et al., 1998). In previous publications, the crystal structures of metal complexes of diclofenac have been reported (Caglar et al., 2013, 2014; Ali \& Jabali, 2016; Dimiza et al., 2011; KovalaDemertzi et al., 1997; Castellari et al., 1999; Kourkoumelis et al., 2004) and in addition its molecular structure has been characterized by various techniques (Iliescu et al., 2004). Based on the above-mentioned points, we report herein the synthesis and structural characterization of a new mixedligand silver(I) complex with dicl and pyrimidine (pym), namely $[\operatorname{Ag}(\mu \text {-dicl })(\mu \text {-pym })]_{n}$, (I).

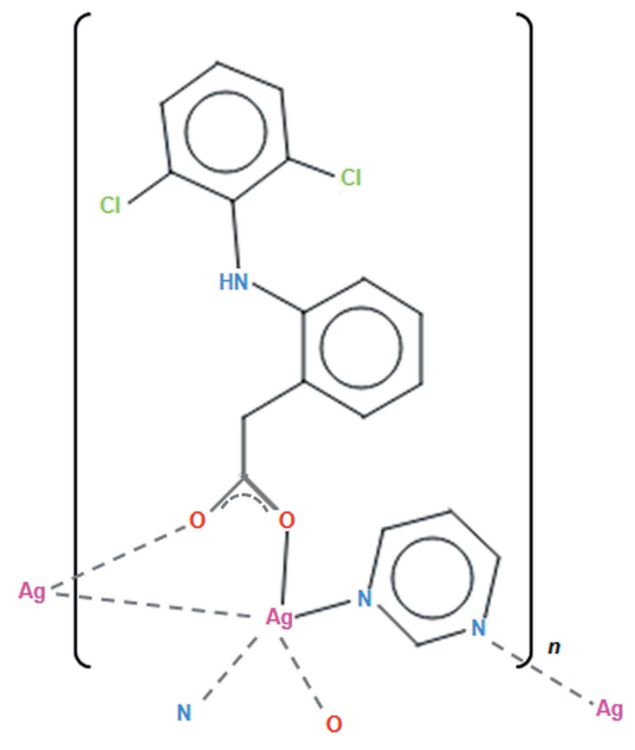

\section{Structural commentary}

In (I), Ag1 atoms are four-coordinated by two carboxylate oxygen atoms [O 2 and $\mathrm{O} 1^{\mathrm{i}}$; symmetry code: (i) $-x+1,-y+1$,

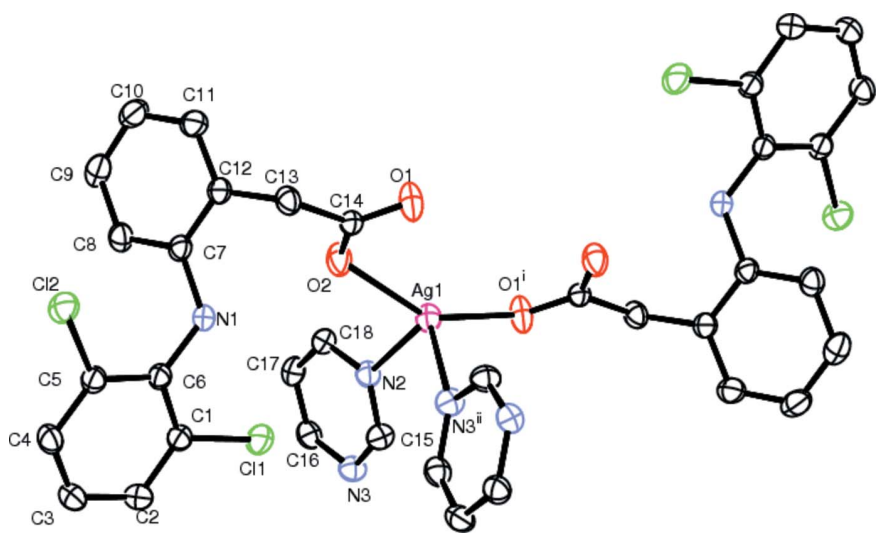

Figure 1

The molecular configuration and atom-labelling scheme for the title complex, (I), with displacement ellipsoids drawn at the $30 \%$ level. For symmetry codes (i) and (ii), see Table 1.
Table 1

Selected geometric parameters $\left(\AA,^{\circ}\right)$.

\begin{tabular}{lclr}
\hline $\mathrm{Ag} 1-\mathrm{O} 2$ & $2.279(2)$ & $\mathrm{Ag} 1-\mathrm{N} 3^{\mathrm{ii}}$ & $2.412(3)$ \\
$\mathrm{Ag} 1-\mathrm{O} 1^{\mathrm{i}}$ & $2.280(2)$ & $\mathrm{Ag} 1-\mathrm{Ag} 1^{\mathrm{i}}$ & $2.8931(5)$ \\
$\mathrm{Ag} 1-\mathrm{N} 2$ & $2.381(3)$ & & \\
& & & \\
$\mathrm{O} 2-\mathrm{Ag} 1-\mathrm{O} 1^{\mathrm{i}}$ & $148.04(10)$ & $\mathrm{O} 2-\mathrm{Ag} 1-\mathrm{Ag} 1^{\mathrm{i}}$ & $81.70(6)$ \\
$\mathrm{O} 2-\mathrm{Ag} 1-\mathrm{N} 2$ & $99.71(8)$ & $\mathrm{O} 1^{\mathrm{i}}-\mathrm{Ag} 1-\mathrm{Ag} 1^{\mathrm{i}}$ & $76.19(6)$ \\
$\mathrm{O} 1^{\mathrm{i}}-\mathrm{Ag} 1-\mathrm{N} 2$ & $89.58(8)$ & $\mathrm{N} 2-\mathrm{Ag} 1-\mathrm{Ag} 1^{\mathrm{i}}$ & $151.80(6)$ \\
$\mathrm{O}^{\mathrm{i}}-\mathrm{Ag} 1-\mathrm{N} 3^{\mathrm{ii}}$ & $108.69(9)$ & $\mathrm{N3}^{\mathrm{ii}}-\mathrm{Ag} 1-\mathrm{Ag} 1^{\mathrm{i}}$ & $99.73(6)$ \\
$\mathrm{N}^{\mathrm{ii}}-\mathrm{Ag} 1-\mathrm{N}^{\mathrm{ii}}$ & $107.93(9)$ & & \\
\hline
\end{tabular}

Symmetry codes: (i) $-x+1,-y+1,-z+2$; (ii) $x,-y+\frac{1}{2}, z+\frac{1}{2}$.

$-z+2$ ] from separate dicl ligands and two nitrogen atoms [N2 and $\mathrm{N} 3{ }^{\text {ii }}$; symmetry code: (ii) $\left.x,-y+\frac{1}{2},-z+\frac{1}{2}\right]$ from two separate pym ligands (Fig. 1). The discrimination parameter for the $\mathrm{AgN}_{2} \mathrm{O}_{2}$ core $\left\{\tau_{4}=\left[\left(360^{\circ}-(\alpha+\beta)\right] / 141^{\circ}\right\}\right.$, where $\alpha$ and $\beta$ are the largest angles around the metal atom) is 0.732 and indicates substantial deviation from ideal tetrahedral geometry (Yang et al., 2007). The $\mathrm{Ag}-\mathrm{N}$ bond lengths [2.381 (3) and 2.412 (3) ̊] (Table 1) are similar to those found in the polymeric mixed-ligand silver(I) complex with 3,5pyridinedicarboxylate (pydc) and (pym), $\left[\operatorname{Ag}_{4}(\mu \text {-pydc })_{2}(\mu\right.$ pym $\left.)_{2}\right]_{n}[2.313$ (5), 2.436 (5) and 2.490 (5) A; Hamamci Alisir et al., 2015). The $\mathrm{Ag}-\mathrm{O}$ bond lengths in (I) [2.279 (2) and 2.280 (2) $\AA$ ] are longer than those in $\left[\mathrm{Ag}_{2}(\mathrm{sal})_{2}\right]_{n}$ (sal = salicylate; 2.1887-2.2043 Aं; Azócar et al., 2013) but shorter than those found in other silver carboxylate complexes (Wu \& Mak, 1995; Zhang et al., 2015; Olson et al., 2006). Each pair of silver(I) atoms in the title complex is bridged by the $\mu_{2^{-}}$ carboxylato- $O, O^{\prime}$ groups of dicl, forming centrosymmetric

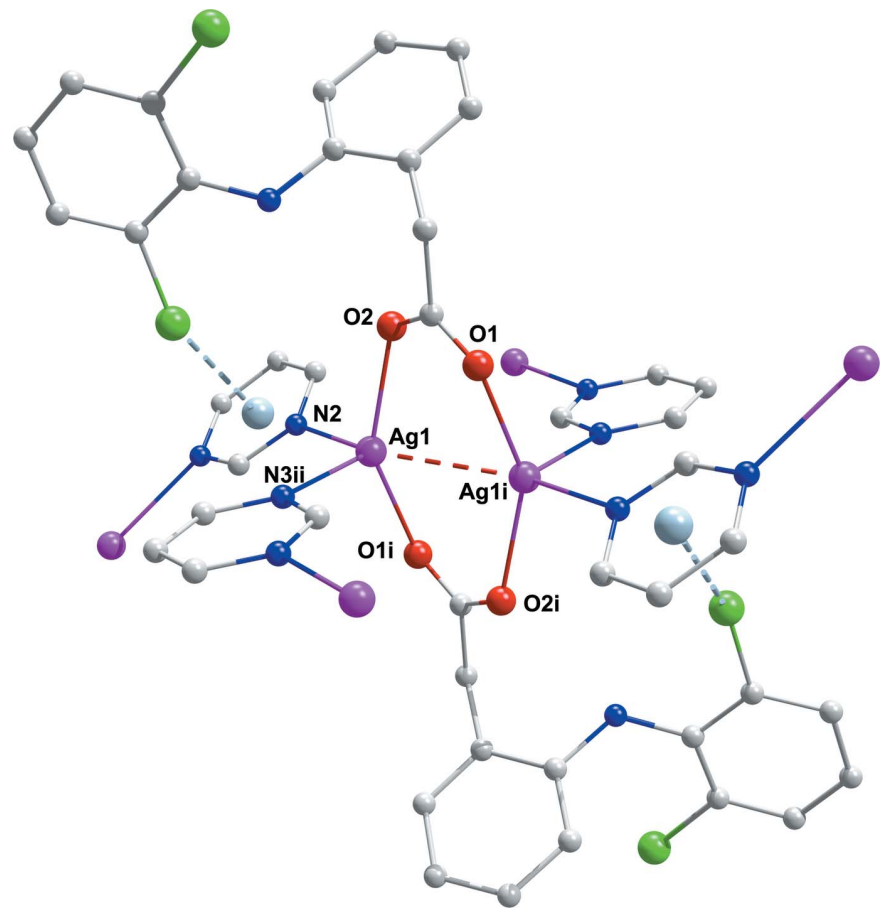

Figure 2

A view of the centrosymmetric caboxylate-bridged dinuclear $\left[\mathrm{Ag}_{2}(\mu\right.$ dicl $)_{2}$ ] unit in (I). $\mathrm{H}$ atoms have been omitted. 
Table 2

Hydrogen-bond geometry $\left(\AA,^{\circ}\right)$.

\begin{tabular}{lllll}
\hline$D-\mathrm{H} \cdots A$ & $D-\mathrm{H}$ & $\mathrm{H} \cdots A$ & $D \cdots A$ & $D-\mathrm{H} \cdots A$ \\
\hline $\mathrm{N} 1-\mathrm{H} 1 \cdots \mathrm{O} 2$ & 0.86 & 2.43 & $2.971(3)$ & 122 \\
$\mathrm{C} 16-\mathrm{H} 16 \cdots \mathrm{O} 1^{\mathrm{iii}}$ & 0.93 & 2.51 & $3.248(4)$ & 136 \\
$\mathrm{C} 13-\mathrm{H} 13 B \cdots C g 6^{\mathrm{iv}}$ & 0.97 & 3.30 & $3.983(3)$ & 129 \\
\hline
\end{tabular}

Symmetry codes: (iii) $x, y, z-1$; (iv) $x,-y+\frac{1}{2}, z-\frac{1}{2}$.

dinuclear $\left[\mathrm{Ag}_{2}(\mu \text {-dicl })_{2}\right]$ units (Fig. 2). Within the units are short intraligand $\mathrm{C} 1-\mathrm{Cl} 1 \cdots \pi$ interactions to the pym ligands [3.6409(15) $\AA]$. The Ag1 $\cdots A g 1^{i}$ separation in the unit [2.8931 (5) $\AA$ ] is significantly shorter than the sum of the van der Waals radii for two silver atoms (3.44 $\AA$ ), indicating weak interactions between adjacent $\mathrm{Ag}^{\mathrm{I}}$ ions, forming an $\left[\mathrm{Ag}_{2}(\mathrm{COO})_{2}\right]$ units. If coexisting strong argentophilic $\mathrm{Ag} 1 \cdots \mathrm{Ag} 1^{\mathrm{i}}$ interactions are considered as coordinative, it could be reasoned that the coordination around Ag1 is slightly distorted trigonal-bipyramidal [the structural distortion index tau $(\tau)$ was calculated to be 0.06] (Addison et al., 1984).

As illustrated in Fig. 3, in the title complex, the pym ligand acts as a $\mu_{2}-N, N^{l}$-bridging ligand between neighboring $\left[\mathrm{Ag}_{2}(\mathrm{COO})_{2}\right]$ units, leading to the formation of a twodimensional coordination polymer, extending along (100) (Fig. 4). In other words, $\left[\mathrm{Ag}_{2}(\mathrm{COO})_{2}\right]$ units, which comprise

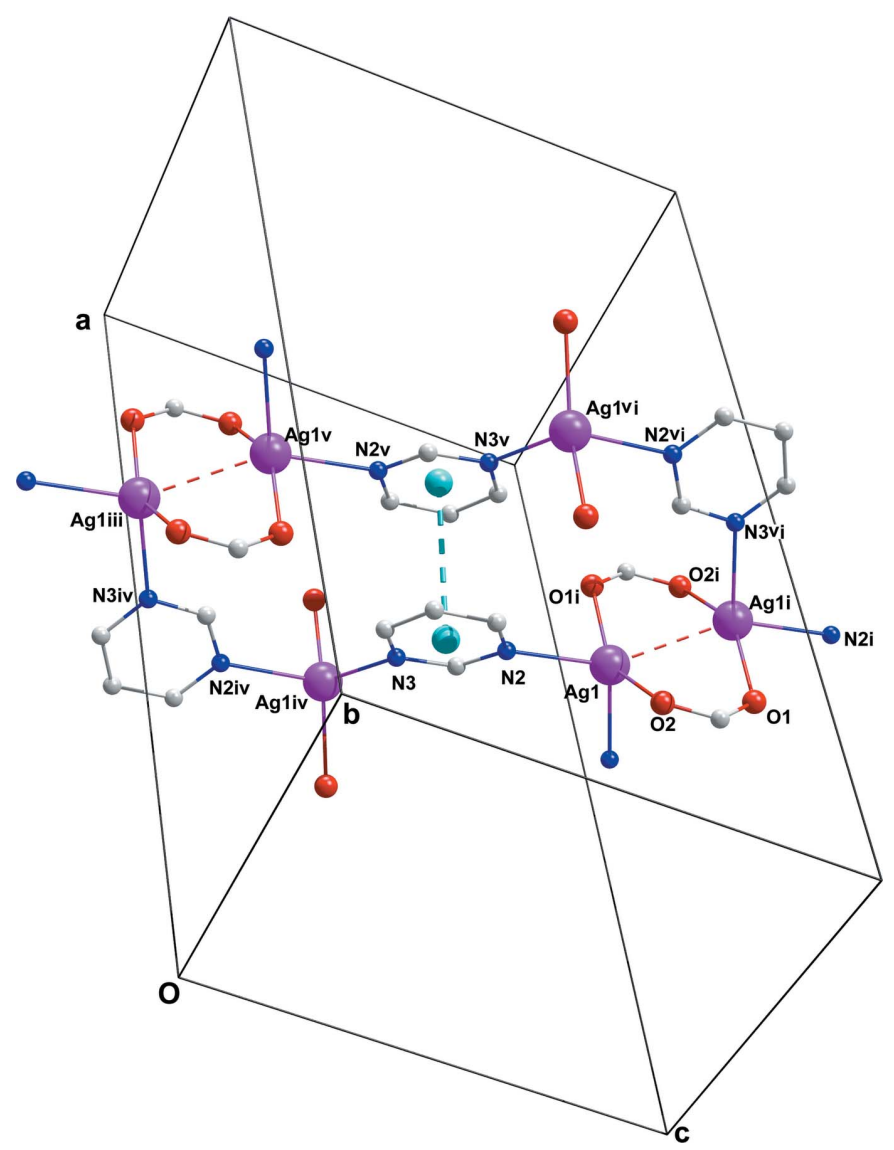

Figure 3

A partial expansion of the dinuclear unit in (I) through the pym ligands, also showing the pym $\cdots$ pym $\pi-\pi$ ring interactions.

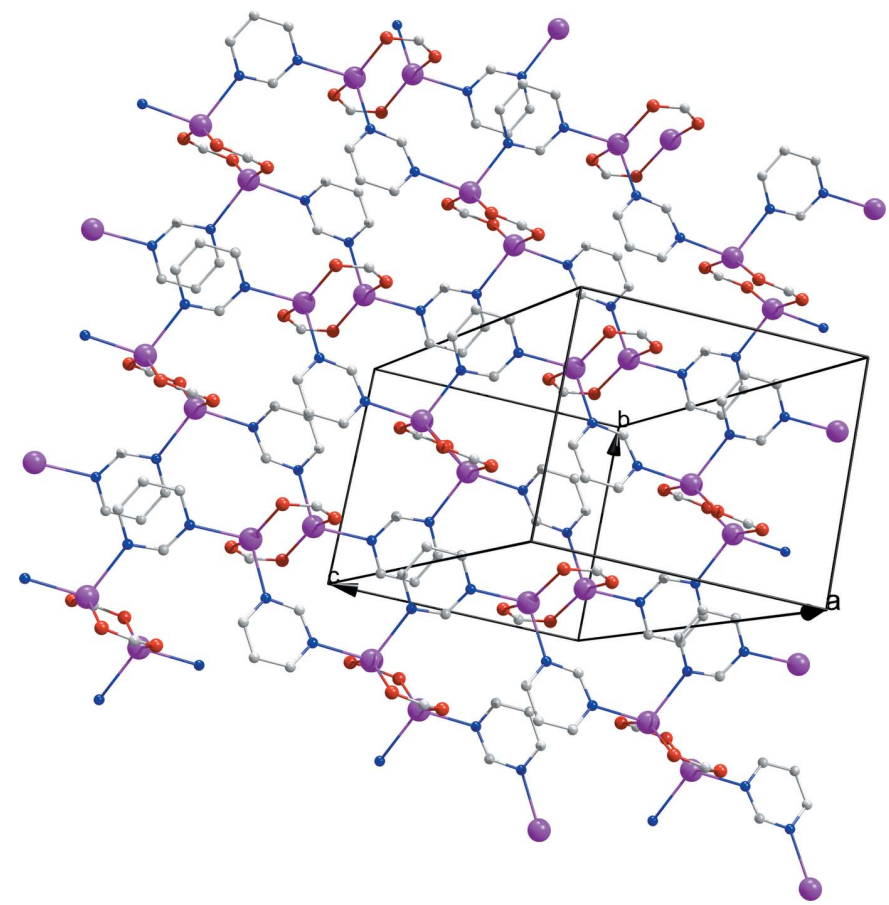

Figure 4

The layered structure of (I). $\mathrm{H}$ atoms and part of the dicl ligands have been omitted.

eight-membered rings, can be defined as the nodes of the structure. Connection of the four different pym ligands to these nodes provides continuity of the structure (Fig. 4).

In the dicl ligand, the two benzene rings form a dihedral angle of $61.42(5)^{\circ}$, the conformation of the ligand being stabilized by an intramolecular $\mathrm{N} 1-\mathrm{H} 1 \cdots \mathrm{O} 2_{\text {carboxyl }}$ hydrogen-bonding interaction [2.971 (3) ̊] (Table 2).

\section{Supramolecular features}

In the crystal, a $\mathrm{C} 16-\mathrm{H} 16 \cdots \mathrm{O} 1^{\mathrm{iii}}$ hydrogen-bonding interaction stabilizes the crystal packing (Table 2). In addition, there is a weak $\mathrm{C} 13-\mathrm{H} 13 \cdots \mathrm{Cg} 6^{\text {iv }}$ interaction to a pym ring [3.983 $\AA$ ] and a strong $\pi-\pi$ stacking interaction between

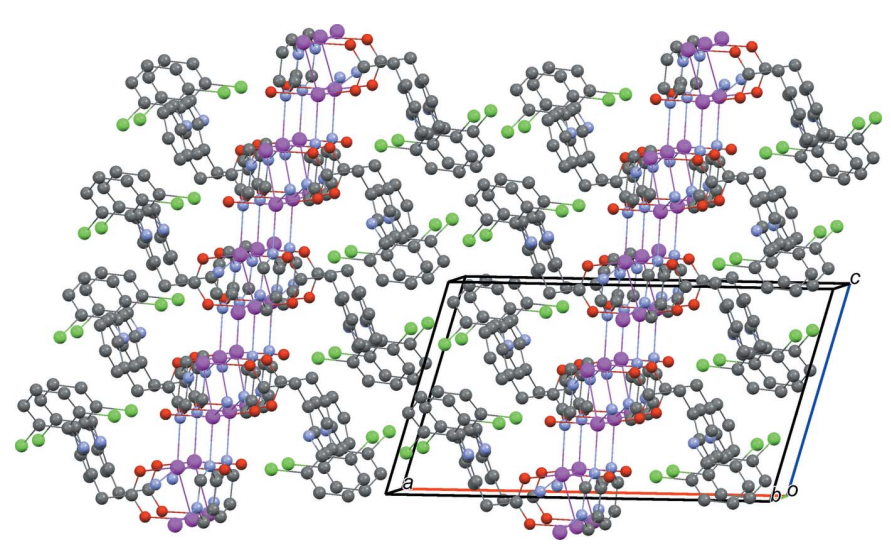

Figure 5

The packing of (I) in the unit cell viewed along the $b$ axis. 
Table 3

Selected comparative IR spectral data for Nadicl and the dicl ligand in (I).

Frequencies in $\mathrm{cm}^{-1} ; w$, weak; $m$, medium; $s$, strong; $v s$, very strong. Nadicl $=$ sodium 2-(2,6-dichloroanilino)phenylacetate.

\begin{tabular}{lll}
\hline Assignment & Nadicl & $(\mathrm{I})$ \\
\hline$v(\mathrm{NH})$ & $3250(m)$ & $3307(m)$ \\
$v_{\mathrm{ar}}(\mathrm{CH})$ & $3060(v w)$ & $3064-3029(v w)$ \\
$\nu_{\mathrm{al}}(\mathrm{CH})$ & $2980(v w)$ & $2956-2890(v w)$ \\
$v_{\mathrm{as}}(\mathrm{COO})$ & $1572(v s)$ & $1548(v s)$ \\
$v_{\mathrm{s}}(\mathrm{COO})$ & $1399(w)$ & $1365(v s)$ \\
$\nu(\mathrm{CCl})$ & $768(s)$ & $768(v s)$ \\
\hline
\end{tabular}

aromatic rings of the pym ligands $\left[\mathrm{Cg} 3 \cdots C g 3^{\mathrm{v}}=\right.$ 3.4199 (17) $\AA$; $C g 3$ is the centroid of the N2/C15/N3/C216-C18 ring; symmetry code $(\mathrm{v}):-x+1,-y+1,-z+1]$, shown in Fig. 3. These interactions are significant for holding layers together in the solid state and generating an overall threedimensional framework structure (Fig. 5).

\section{Synthesis and crystallization}

All reactions were performed with commercially available reagents and used without further purification. Solid sodium 2-(2,6-dicholoroanilino)phenylacetate (Nadicl) (0.32 g, $1 \mathrm{mmol})$ and pyrimidine $(0.08 \mathrm{~g}, 1 \mathrm{~mol})$ were added to an aqueous solution $\left(10 \mathrm{~cm}^{3}\right)$ of $\mathrm{AgNO}_{3}(0.17 \mathrm{~g}, 1 \mathrm{mmol})$ with stirring. A white suspension with a white precipitate formed and the addition of acetonitrile $\left(10 \mathrm{~cm}^{3}\right)$ to this resulted in a clear solution which was left to stand for slow evaporation in darkness at room temperature. Single crystals of (I) suitable for X-ray analysis were obtained within a few days.

\section{Spectroscopy}

The infrared spectrum was obtained using a Perkin Elmer Spectrum Two FTIR with a diamond Attenuated Total Reflectance attachment (ATR) in the frequency range 4000$600 \mathrm{~cm}^{-1}$. The sample was placed on the ATR crystal and pressure exerted by screwing the pressure clamp onto the sample to ensure maximum contact with the ATR crystal. The characteristic absorption bands of Nadicl and the title complex are listed in Table 3. The spectrum is deposited as a supplementary Fig. S1.

The characteristic absorption band in the FT-IR spectra of the carboxylate complexes is the asymmetric $\left(v_{\mathrm{as}}\right)$ and symmetric $\left(v_{\mathrm{s}}\right)$ vibrations of the carboxylate group. The difference between the asymmetric and symmetric carboxylate stretching $\left[\Delta v=v_{\mathrm{as}}\left(\mathrm{COO}^{-}\right)-v_{\mathrm{s}}\left(\mathrm{COO}^{-}\right)\right]$is often used to correlate the infrared spectra of metal carboxylate structures. When $\Delta v<200 \mathrm{~cm}^{-1}$, the carboxylate groups of the complexes can be considered bidentate (Azócar et al., 2013). The value of $\Delta v$ is calculated as $183 \mathrm{~cm}^{-1}$ for 1 . Based on the above-mentioned points, it is suggested that carboxylate groups in the complex exhibit a bidentate coordination mode, as revealed by the structural analysis.
Table 4

Experimental details.

\begin{tabular}{|c|c|}
\hline \multicolumn{2}{|l|}{ Crystal data } \\
\hline Chemical formula & {$\left[\mathrm{Ag}\left(\mathrm{C}_{14} \mathrm{H}_{10} \mathrm{Cl}_{2} \mathrm{NO}_{2}\right)\left(\mathrm{C}_{4} \mathrm{H}_{4} \mathrm{~N}_{2}\right)\right]$} \\
\hline$M_{\mathrm{r}}$ & 483.09 \\
\hline Crystal system, space group & Monoclinic, $P 2_{1} / c$ \\
\hline Temperature $(\mathrm{K})$ & 293 \\
\hline$a, b, c(\AA)$ & $18.5886(4), 9.3071(4), 10.6646(8)$ \\
\hline$\beta\left({ }^{\circ}\right)$ & $105.644(3)$ \\
\hline$V\left(\mathrm{~A}^{3}\right)$ & $1776.69(16)$ \\
\hline$Z$ & 4 \\
\hline Radiation type & Mo $K \alpha$ \\
\hline$\mu\left(\mathrm{mm}^{-1}\right)$ & 1.45 \\
\hline Crystal size $(\mathrm{mm})$ & $0.60 \times 0.46 \times 0.27$ \\
\hline \multicolumn{2}{|l|}{ Data collection } \\
\hline Diffractometer & Stoe IPDS2 \\
\hline Absorption correction & $\begin{array}{l}\text { Integration ( } X \text {-RED } 32 \text {; Stoe \& Cie, } \\
\text { 2002) }\end{array}$ \\
\hline$T_{\min }, T_{\max }$ & $0.471,0.693$ \\
\hline $\begin{array}{l}\text { No. of measured, independent and } \\
\text { observed }[I>2 \sigma(I)] \text { reflections }\end{array}$ & $13090,4538,3672$ \\
\hline$R_{\text {int }}$ & 0.088 \\
\hline$(\sin \theta / \lambda)_{\max }\left(\AA^{-1}\right)$ & 0.675 \\
\hline \multicolumn{2}{|l|}{ Refinement } \\
\hline$R\left[F^{2}>2 \sigma\left(F^{2}\right)\right], w R\left(F^{2}\right), S$ & $0.039,0.095,1.04$ \\
\hline No. of reflections & 4538 \\
\hline No. of parameters & 236 \\
\hline $\mathrm{H}$-atom treatment & H-atom parameters constrained \\
\hline$\Delta \rho_{\max }, \Delta \rho_{\min }\left(\mathrm{e} \AA^{-3}\right)$ & $0.57,-1.14$ \\
\hline
\end{tabular}

Computer programs: X-AREA and X-RED32 (Stoe \& Cie, 2002), SHELXS97 (Sheldrick, 2008) within WinGX (Farrugia, 2012), SHELXL2014 (Sheldrick, 2015), ORTEP-3 for Windows (Farrugia, 2012) and SHELXTL (Sheldrick, 2008).

\section{Refinement}

Crystal data, data collection and structure refinement details are summarized in Table 4. All C-bound hydrogen atoms in (I) were included in calculated positions with $\mathrm{C}-\mathrm{H}=0.93 \AA$ (aromatic) or $0.97 \AA$ (methylene) and allowed to ride, with $U_{\text {iso }}(\mathrm{H})=1.2 U_{\text {eq }}(\mathrm{C})$. The $\mathrm{N}$-bound $\mathrm{H}$ atom was located in a difference-Fourier map but was also allowed to ride in the refinement with $U_{\text {iso }}(\mathrm{H})=1.2 U_{\text {eq }}(\mathrm{N})$.

\section{References}

Addison, A. W., Rao, T. N., Reedijk, J., van Rijn, J. \& Verschoor, G. C. (1984). J. Chem. Soc. Dalton Trans. pp. 1349-1356.

Ali, H. A. \& Jabali, B. (2016). Polyhedron, 107, 97-106.

Azócar, M., Muñoz, H., Levin, P., Dinamarca, N., Gomez, G., Ibanez, A., Garland, M. T. \& Paez, M. A. (2013). Commun. Inorg. Synth. 1, $19-21$.

Caglar, S., Aydemir, I. E., Adıgüzel, E., Caglar, B., Demir, S. \& Büyükgüngör, O. (2013). Inorg. Chim. Acta, 408, 131-138.

Caglar, S., Aydemir, I. E., Cankaya, M., Kuzucu, M., Temel, E. \& Büyükgüngör, O. (2014). J. Coord. Chem. 67, 969-985.

Castellari, C., Feroci, G. \& Ottani, S. (1999). Acta Cryst. C55, 907-910.

Dimiza, F., Perdih, F., Tangoulis, V., Turel, I., Kessissoglou, D. P. \& Psomas, G. (2011). J. Inorg. Biochem. 105, 476-489.

Duffy, C. P., Elliott, C. J., O'Connor, R. A., Heenan, M. M., Coyle, S., Cleary, I. M., Kavanagh, K., Verhaegen, S., O'Loughlin, C. M., NicAmhlaoibh, R. \& Clynes, M. (1998). Eur. J. Cancer, 34, 12501259.

Farrugia, L. J. (2012). J. Appl. Cryst. 45, 849-854.

Hamamci Alisir, S., Demir, S., Sariboga, B. \& Buyukgungor, O. (2015). J. Coord. Chem. 68, 155-168. 
Iliescu, T., Baia, M. \& Kiefer, W. (2004). Chem. Phys. 298, 167-174.

Kim, K., Yoon, J., Kim, J. K., Baek, S. J., Eling, T. E., Lee, W. J., Ryu, J., Lee, J. G., Lee, J. \& Yoo, J. (2004). Biochem. Biophys. Res. Commun. 325, 1298-1303.

Kourkoumelis, N., Demertzis, M. A., Kovala-Demertzi, D., Koutsodimou, A. \& Moukarika, A. (2004). Spectrochim. Acta Part A, 60, 2253-2259.

Kovala-Demertzi, D., Theodorou, A., Demertzis, M. A., Raptopoulou, C. P. \& Terzis, A. (1997). J. Inorg. Biochem. 65, 151-157.

Njogu, E. M., Omond1, B. \& Nyamorı, V. O. (2015). J. Coord. Chem. 68, 3389-3431.

Nomiya, K., Takahashi, S. \& Noguchi, R. (2000). J. Chem. Soc. Dalton Trans. pp. 2091-2097.

Olson, L., Whitcomb, D. R., Rajeswaran, M., Blanton, T. N. \& Stwertka, B. J. (2006). Chem. Mater. 18, 1667-1674.

Pyykkö, P. (1997). Chem. Rev. 97, 597-636.

Ribeiro, G., Benadiba, M., Colquhoun, A. \& de Oliveira Silva, D. (2008). Polyhedron, 27, 1131-1137.
Sheldrick, G. M. (2008). Acta Cryst. A64, 112-122.

Sheldrick, G. M. (2015). Acta Cryst. C71, 3-8.

Stoe \& Cie (2002). $X$-AREA and $X$-RED32. Stoe \& Cie, Darmstadt, Germany.

Wang, Z. \& Cohen, S. M. (2009). Chem. Soc. Rev. 38, 1315-1329.

Weder, J. E., Dillon, C. T., Hambley, T. W., Kennedy, B. J., Lay, P. A., Biffin, J. R., Regtop, H. L. \& Davies, N. M. (2002). Coord. Chem. Rev. 232, 95-126.

Wu, D. D. \& Mak, T. C. W. (1995). J. Chem. Soc. Dalton Trans. pp. 2671-2678.

Yam, V. W. \& Lo, K. (1999). Chem. Soc. Rev. 28, 323-334.

Yang, L., Powell, D. R. \& Houser, R. P. (2007). Dalton Trans. pp. 955964.

Zhang, J. P., Huang, X. C. \& Chen, X. M. (2009). Chem. Soc. Rev. 38, 2385-2396.

Zhang, T., Huang, H. Q., Mei, H. X., Wang, D. F., Wang, X., Huang, R. \& Zheng, L. (2015). J. Mol. Struct. 1100, 237-244. 


\section{supporting information}

Acta Cryst. (2016). E72, 1475-1479 [https://doi.org/10.1107/S2056989016014730]

\section{Crystal structure of a mixed-ligand silver(I) complex of the non-steroidal anti- inflammatory drug diclofenac and pyrimidine}

\section{Sevim Hamamci Alisir and Necmi Dege}

Computing details

Data collection: $X$-AREA (Stoe \& Cie, 2002); cell refinement: $X$-AREA (Stoe \& Cie, 2002); data reduction: $X$-RED32

(Stoe \& Cie, 2002); program(s) used to solve structure: SHELXS97 (Sheldrick, 2008) within WinGX (Farrugia, 2012); program(s) used to refine structure: SHELXL2014 (Sheldrick, 2015); molecular graphics: ORTEP-3 for Windows (Farrugia, 2012); software used to prepare material for publication: SHELXTL (Sheldrick, 2008).

Poly $\left[\left\{\mu_{2}-2-\left[2-\left(2,6-\right.\right.\right.\right.$ dichloroanilino) phenyl]acetato- $\left.\kappa^{2} O: O^{\prime}\right\}\left(\mu_{2}-\right.$ pyrimidine- $\left.\left.\kappa^{2} N^{1}: N^{3}\right) \operatorname{silver}(\mathrm{I})\right]$

Crystal data

$\left[\mathrm{Ag}\left(\mathrm{C}_{14} \mathrm{H}_{10} \mathrm{Cl}_{2} \mathrm{NO}_{2}\right)\left(\mathrm{C}_{4} \mathrm{H}_{4} \mathrm{~N}_{2}\right)\right]$

$M_{r}=483.09$

Monoclinic, $P 2{ }_{1} / C$

$a=18.5886(4) \AA$

$b=9.3071(4) \AA$

$c=10.6646(8) \AA$

$\beta=105.644(3)^{\circ}$

$V=1776.69(16) \AA^{3}$

$Z=4$

Data collection

Stoe IPDS2 diffractometer $\omega$-scan rotation method Absorption correction: integration (X-RED32; Stoe \& Cie, 2002)

$T_{\min }=0.471, T_{\max }=0.693$

13090 measured reflections

\section{Refinement}

Refinement on $F^{2}$

Least-squares matrix: full

$R\left[F^{2}>2 \sigma\left(F^{2}\right)\right]=0.039$

$w R\left(F^{2}\right)=0.095$

$S=1.04$

4538 reflections

236 parameters

0 restraints

Hydrogen site location: inferred from neighbouring sites
$F(000)=960$

$D_{\mathrm{x}}=1.806 \mathrm{Mg} \mathrm{m}^{-3}$

Mo $K \alpha$ radiation, $\lambda=0.71073 \AA$

Cell parameters from 13681 reflections

$\theta=2.0-29.1^{\circ}$

$\mu=1.45 \mathrm{~mm}^{-1}$

$T=293 \mathrm{~K}$

Prism, colorless

$0.60 \times 0.46 \times 0.27 \mathrm{~mm}$

4538 independent reflections

3672 reflections with $I>2 \sigma(I)$

$R_{\text {int }}=0.088$

$\theta_{\max }=28.7^{\circ}, \theta_{\min }=2.3^{\circ}$

$h=-24 \rightarrow 24$

$k=-12 \rightarrow 12$

$l=-14 \rightarrow 14$

$\mathrm{H}$-atom parameters constrained

$w=1 /\left[\sigma^{2}\left(F_{\mathrm{o}}{ }^{2}\right)+(0.0488 P)^{2}\right]$

where $P=\left(F_{\mathrm{o}}{ }^{2}+2 F_{\mathrm{c}}{ }^{2}\right) / 3$

$(\Delta / \sigma)_{\max }=0.001$

$\Delta \rho_{\max }=0.57 \mathrm{e} \AA^{-3}$

$\Delta \rho_{\min }=-1.14$ e $\AA^{-3}$

Extinction correction: SHELXL2014

(Sheldrick, 2015),

$\mathrm{Fc}^{*}=\mathrm{kFc}\left[1+0.001 \mathrm{xFc}^{2} \lambda^{3} / \sin (2 \theta)\right]^{-1 / 4}$

Extinction coefficient: 0.0206 (11) 


\section{Special details}

Geometry. All esds (except the esd in the dihedral angle between two 1.s. planes) are estimated using the full covariance matrix. The cell esds are taken into account individually in the estimation of esds in distances, angles and torsion angles; correlations between esds in cell parameters are only used when they are defined by crystal symmetry. An approximate (isotropic) treatment of cell esds is used for estimating esds involving l.s. planes.

Fractional atomic coordinates and isotropic or equivalent isotropic displacement parameters $\left(\hat{A}^{2}\right)$

\begin{tabular}{|c|c|c|c|c|}
\hline & $x$ & $y$ & $z$ & $U_{\text {iso }} * / U_{\text {eq }}$ \\
\hline $\mathrm{C} 1$ & $0.17222(14)$ & $0.3200(3)$ & $0.6246(3)$ & $0.0419(6)$ \\
\hline $\mathrm{C} 2$ & $0.12206(16)$ & $0.2364(3)$ & $0.5353(3)$ & $0.0496(7)$ \\
\hline $\mathrm{H} 2$ & 0.1387 & 0.1695 & 0.4847 & $0.060^{*}$ \\
\hline $\mathrm{C} 3$ & $0.04629(16)$ & $0.2541(4)$ & $0.5223(4)$ & $0.0546(8)$ \\
\hline H3 & 0.0116 & 0.2009 & 0.4607 & $0.065^{*}$ \\
\hline $\mathrm{C} 4$ & $0.02263(15)$ & 0.3495 (4) & 0.5995 (3) & $0.0505(7)$ \\
\hline $\mathrm{H} 4$ & -0.0282 & 0.3603 & 0.5912 & $0.061 *$ \\
\hline $\mathrm{C} 5$ & $0.07381(14)$ & $0.4302(3)$ & $0.6904(3)$ & $0.0406(6)$ \\
\hline C6 & $0.15110(13)$ & $0.4220(3)$ & 0.7029 (3) & $0.0381(5)$ \\
\hline $\mathrm{C} 7$ & $0.19762(12)$ & $0.6542(3)$ & 0.8068 (3) & $0.0377(5)$ \\
\hline $\mathrm{C} 8$ & $0.15520(14)$ & $0.7377(3)$ & 0.7067 (3) & $0.0451(6)$ \\
\hline H8 & 0.1312 & 0.6953 & 0.6275 & $0.054^{*}$ \\
\hline C9 & $0.14820(16)$ & 0.8839 (4) & $0.7235(4)$ & $0.0529(8)$ \\
\hline H9 & 0.1197 & 0.9397 & 0.6558 & $0.063^{*}$ \\
\hline $\mathrm{C} 10$ & 0.18355 (17) & $0.9463(3)$ & 0.8408 (4) & $0.0573(9)$ \\
\hline H10 & 0.1785 & 1.0443 & 0.8535 & $0.069^{*}$ \\
\hline C11 & $0.22657(15)$ & 0.8624 (3) & $0.9396(4)$ & $0.0501(7)$ \\
\hline H11 & 0.2502 & 0.9054 & 1.0187 & $0.060 *$ \\
\hline $\mathrm{C} 12$ & $0.23575(12)$ & $0.7165(3)$ & 0.9249 (3) & $0.0391(6)$ \\
\hline $\mathrm{C} 13$ & $0.28437(13)$ & 0.6294 (4) & $1.0340(3)$ & $0.0437(6)$ \\
\hline H13A & 0.2575 & 0.5429 & 1.0448 & $0.052 *$ \\
\hline H13B & 0.2930 & 0.6843 & 1.1140 & $0.052^{*}$ \\
\hline C14 & $0.36042(13)$ & 0.5857 (3) & 1.0147 (3) & $0.0367(5)$ \\
\hline $\mathrm{C} 15$ & $0.44153(16)$ & 0.3069 (3) & $0.5812(3)$ & $0.0444(6)$ \\
\hline H15 & 0.4577 & 0.2251 & 0.6310 & $0.053 *$ \\
\hline $\mathrm{C} 16$ & $0.40626(15)$ & $0.4123(3)$ & 0.3828 (3) & $0.0461(6)$ \\
\hline H16 & 0.3968 & 0.4080 & 0.2926 & $0.055^{*}$ \\
\hline $\mathrm{C} 17$ & $0.39630(16)$ & 0.5399 (4) & 0.4394 (3) & $0.0489(7)$ \\
\hline H17 & 0.3807 & 0.6220 & 0.3899 & $0.059^{*}$ \\
\hline $\mathrm{C} 18$ & $0.41040(15)$ & 0.5417 (3) & $0.5740(3)$ & $0.0461(6)$ \\
\hline H18 & 0.4038 & 0.6266 & 0.6154 & $0.055^{*}$ \\
\hline Ag1 & $0.46373(2)$ & $0.43353(3)$ & $0.87652(2)$ & $0.04354(10)$ \\
\hline Cl1 & $0.26694(4)$ & $0.29612(9)$ & $0.63922(10)$ & $0.0610(2)$ \\
\hline $\mathrm{Cl} 2$ & 0.03984 (4) & $0.54045(9)$ & $0.79226(9)$ & $0.05280(19)$ \\
\hline N1 & $0.20327(12)$ & $0.5025(3)$ & $0.7931(3)$ & $0.0443(5)$ \\
\hline $\mathrm{H} 1$ & 0.2409 & 0.4588 & 0.8434 & $0.053^{*}$ \\
\hline $\mathrm{N} 2$ & $0.43318(12)$ & $0.4245(3)$ & $0.6450(2)$ & $0.0425(5)$ \\
\hline N3 & $0.42916(13)$ & 0.2935 (3) & $0.4527(2)$ & $0.0457(5)$ \\
\hline $\mathrm{O} 1$ & $0.41252(11)$ & $0.5735(3)$ & $1.1157(2)$ & $0.0586(6)$ \\
\hline
\end{tabular}




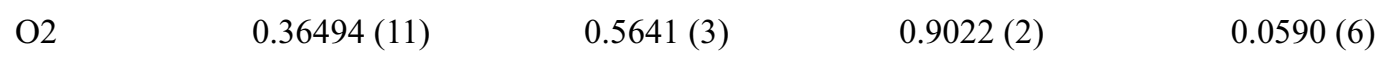

Atomic displacement parameters $\left(\AA^{2}\right)$

\begin{tabular}{|c|c|c|c|c|c|c|}
\hline & $U^{11}$ & $U^{22}$ & $U^{33}$ & $U^{12}$ & $U^{13}$ & $U^{23}$ \\
\hline $\mathrm{C} 1$ & $0.0374(11)$ & $0.0372(13)$ & $0.0497(15)$ & $0.0009(10)$ & $0.0092(11)$ & $0.0018(13)$ \\
\hline $\mathrm{C} 2$ & $0.0522(15)$ & $0.0425(15)$ & $0.0518(17)$ & $-0.0023(12)$ & $0.0097(13)$ & $-0.0065(14)$ \\
\hline $\mathrm{C} 3$ & $0.0458(14)$ & $0.0469(16)$ & $0.063(2)$ & $-0.0076(12)$ & $0.0001(13)$ & $-0.0057(16)$ \\
\hline $\mathrm{C} 4$ & $0.0350(12)$ & $0.0496(17)$ & $0.062(2)$ & $-0.0056(11)$ & $0.0045(12)$ & $0.0027(15)$ \\
\hline $\mathrm{C} 5$ & $0.0362(11)$ & $0.0378(13)$ & $0.0472(15)$ & $0.0012(10)$ & 0.0104 (11) & $0.0046(12)$ \\
\hline C6 & $0.0341(10)$ & $0.0344(12)$ & $0.0430(14)$ & $0.0025(9)$ & $0.0055(10)$ & $0.0047(11)$ \\
\hline $\mathrm{C} 7$ & $0.0293(10)$ & $0.0376(13)$ & $0.0472(14)$ & $0.0020(9)$ & $0.0119(10)$ & $0.0004(12)$ \\
\hline $\mathrm{C} 8$ & $0.0339(11)$ & $0.0495(15)$ & $0.0504(16)$ & $0.0006(11)$ & $0.0090(11)$ & $0.0030(14)$ \\
\hline C9 & $0.0402(13)$ & $0.0480(16)$ & $0.072(2)$ & $0.0062(12)$ & $0.0182(14)$ & $0.0166(17)$ \\
\hline $\mathrm{C} 10$ & $0.0468(15)$ & $0.0384(15)$ & 0.089 & $0.0036(12)$ & $0.0230(16)$ & $-0.0026(17)$ \\
\hline $\mathrm{C} 11$ & $0.0383(12)$ & $0.0468(16)$ & $0.067(2)$ & $-0.0010(11)$ & $0.0167(13)$ & $-0.0129(15)$ \\
\hline $\mathrm{C} 12$ & $0.0267(10)$ & $0.0422(14)$ & $0.0502(15)$ & $0.0005(9)$ & $0.0135(10)$ & $-0.0046(12)$ \\
\hline $\mathrm{C} 13$ & $0.0319(11)$ & $0.0561(17)$ & $0.0432(15)$ & $0.0005(11)$ & $0.0102(10)$ & $-0.0061(14)$ \\
\hline $\mathrm{C} 14$ & $0.0303(10)$ & $0.0338(12)$ & $0.0445(14)$ & $0.0008(9)$ & $0.0074(10)$ & $-0.0019(11)$ \\
\hline $\mathrm{C} 15$ & $0.0539(14)$ & $0.0419(14)$ & $0.0395(14)$ & $0.0020(12)$ & $0.0159(12)$ & $0.0026(13)$ \\
\hline $\mathrm{C} 16$ & $0.0441(13)$ & $0.0577(18)$ & $0.0356(13)$ & $-0.0024(12)$ & $0.0094(11)$ & $0.0018(13)$ \\
\hline $\mathrm{C} 17$ & $0.0451(13)$ & $0.0503(16)$ & $0.0493(16)$ & $0.0086(12)$ & $0.0096(12)$ & $0.0110(14)$ \\
\hline $\mathrm{C} 18$ & $0.0404(12)$ & $0.0432(15)$ & $0.0522(17)$ & $0.0056(11)$ & $0.0084(12)$ & $-0.0035(13)$ \\
\hline Ag1 & $0.04020(13)$ & $0.05477(15)$ & $0.03712(13)$ & $0.00513(8)$ & $0.01297(8)$ & $-0.00042(10)$ \\
\hline $\mathrm{Cl} 1$ & $0.0401(3)$ & $0.0555(4)$ & $0.0877(6)$ & $0.0068(3)$ & $0.0176(3)$ & $-0.0087(5)$ \\
\hline $\mathrm{Cl} 2$ & $0.0458(3)$ & $0.0534(4)$ & $0.0647(5)$ & $0.0013(3)$ & $0.0243(3)$ & $-0.0020(4)$ \\
\hline N1 & $0.0344(10)$ & $0.0402(12)$ & $0.0504(14)$ & $0.0065(9)$ & $-0.0019(9)$ & $-0.0026(11)$ \\
\hline $\mathrm{N} 2$ & $0.0401(10)$ & $0.0500(14)$ & $0.0375(11)$ & $0.0031(9)$ & $0.0104(9)$ & $-0.0026(11)$ \\
\hline N3 & $0.0534(13)$ & $0.0460(13)$ & $0.0392(12)$ & $-0.0014(10)$ & $0.0149(10)$ & $-0.0015(11)$ \\
\hline $\mathrm{O} 1$ & $0.0334(9)$ & 0.0967 (19) & $0.0426(11)$ & $0.0109(10)$ & $0.0049(8)$ & $0.0071(12)$ \\
\hline $\mathrm{O} 2$ & $0.0412(10)$ & $0.0880(18)$ & $0.0456(12)$ & $0.0186(10)$ & $0.0080(9)$ & $-0.0133(12)$ \\
\hline
\end{tabular}

Geometric parameters $\left(\AA,{ }^{\circ}\right)$

\begin{tabular}{llll}
\hline $\mathrm{C} 1-\mathrm{C} 2$ & $1.379(4)$ & $\mathrm{C} 12-\mathrm{C} 13$ & $1.504(4)$ \\
$\mathrm{C} 1-\mathrm{C} 6$ & $1.390(4)$ & $\mathrm{C} 13-\mathrm{C} 14$ & $1.537(3)$ \\
$\mathrm{C} 1-\mathrm{C} 11$ & $1.739(3)$ & $\mathrm{C} 13-\mathrm{H} 13 \mathrm{~A}$ & 0.9700 \\
$\mathrm{C} 2-\mathrm{C} 3$ & $1.387(4)$ & $\mathrm{C} 13-\mathrm{H} 13 \mathrm{~B}$ & $1.242(4)$ \\
$\mathrm{C} 2-\mathrm{H} 2$ & 0.9300 & $\mathrm{C} 14-\mathrm{O} 2$ & $1.244(3)$ \\
$\mathrm{C} 3-\mathrm{C} 4$ & $1.362(5)$ & $\mathrm{C} 14-\mathrm{O} 1$ & $1.320(4)$ \\
$\mathrm{C} 3-\mathrm{H} 3$ & 0.9300 & $\mathrm{C} 15-\mathrm{N} 2$ & $1.333(4)$ \\
$\mathrm{C} 4-\mathrm{C} 5$ & $1.382(4)$ & $\mathrm{C} 15-\mathrm{N} 3$ & 0.9300 \\
$\mathrm{C} 4-\mathrm{H} 4$ & 0.9300 & $\mathrm{C} 15-\mathrm{H} 15$ & $1.338(4)$ \\
$\mathrm{C} 5-\mathrm{C} 6$ & $1.409(3)$ & $\mathrm{C} 16-\mathrm{N} 3$ & $1.367(5)$ \\
$\mathrm{C} 5-\mathrm{C} 2$ & $1.733(3)$ & $\mathrm{C} 16-\mathrm{C} 17$ & 0.9300 \\
$\mathrm{C} 6-\mathrm{N} 1$ & $\mathrm{C} 16-\mathrm{H} 16$ & $1.388(5)$ \\
$\mathrm{C} 7-\mathrm{C} 8$ & $1.387(3)$ & $\mathrm{C} 17-\mathrm{C} 18$ & 0.9300 \\
$\mathrm{C} 7-\mathrm{C} 12$ & $1.382(4)$ & $\mathrm{C} 17-\mathrm{H} 17$ &
\end{tabular}




\begin{tabular}{|c|c|c|c|}
\hline $\mathrm{C} 7-\mathrm{N} 1$ & $1.426(4)$ & $\mathrm{C} 18-\mathrm{N} 2$ & $1.330(4)$ \\
\hline $\mathrm{C} 8-\mathrm{C} 9$ & $1.383(5)$ & $\mathrm{C} 18-\mathrm{H} 18$ & 0.9300 \\
\hline $\mathrm{C} 8-\mathrm{H} 8$ & 0.9300 & $\mathrm{Ag} 1-\mathrm{O} 2$ & $2.279(2)$ \\
\hline $\mathrm{C} 9-\mathrm{C} 10$ & $1.375(6)$ & $\mathrm{Ag} 1-\mathrm{O} 1^{\mathrm{i}}$ & $2.280(2)$ \\
\hline C9-H9 & 0.9300 & $\mathrm{Ag} 1-\mathrm{N} 2$ & $2.381(3)$ \\
\hline $\mathrm{C} 10-\mathrm{C} 11$ & $1.380(5)$ & $\mathrm{Ag} 1-\mathrm{N} 3^{\mathrm{ii}}$ & $2.412(3)$ \\
\hline $\mathrm{C} 10-\mathrm{H} 10$ & 0.9300 & $\mathrm{Ag} 1-\mathrm{Ag} 1^{\mathrm{i}}$ & $2.8931(5)$ \\
\hline $\mathrm{C} 11-\mathrm{C} 12$ & $1.383(4)$ & $\mathrm{N} 1-\mathrm{H} 1$ & 0.8600 \\
\hline C11-H11 & 0.9300 & & \\
\hline $\mathrm{C} 2-\mathrm{C} 1-\mathrm{C} 6$ & $123.5(2)$ & $\mathrm{C} 12-\mathrm{C} 13-\mathrm{H} 13 \mathrm{~B}$ & 108.5 \\
\hline $\mathrm{C} 2-\mathrm{C} 1-\mathrm{Cl} 1$ & $118.0(2)$ & $\mathrm{C} 14-\mathrm{C} 13-\mathrm{H} 13 \mathrm{~B}$ & 108.5 \\
\hline $\mathrm{C} 6-\mathrm{C} 1-\mathrm{Cl1}$ & $118.5(2)$ & $\mathrm{H} 13 \mathrm{~A}-\mathrm{C} 13-\mathrm{H} 13 \mathrm{~B}$ & 107.5 \\
\hline $\mathrm{C} 1-\mathrm{C} 2-\mathrm{C} 3$ & $118.8(3)$ & $\mathrm{O} 2-\mathrm{C} 14-\mathrm{O} 1$ & $125.6(2)$ \\
\hline $\mathrm{C} 1-\mathrm{C} 2-\mathrm{H} 2$ & 120.6 & $\mathrm{O} 2-\mathrm{C} 14-\mathrm{C} 13$ & $118.5(2)$ \\
\hline $\mathrm{C} 3-\mathrm{C} 2-\mathrm{H} 2$ & 120.6 & $\mathrm{O} 1-\mathrm{C} 14-\mathrm{C} 13$ & $115.9(3)$ \\
\hline $\mathrm{C} 4-\mathrm{C} 3-\mathrm{C} 2$ & $120.1(3)$ & $\mathrm{N} 2-\mathrm{C} 15-\mathrm{N} 3$ & $126.6(3)$ \\
\hline $\mathrm{C} 4-\mathrm{C} 3-\mathrm{H} 3$ & 119.9 & $\mathrm{~N} 2-\mathrm{C} 15-\mathrm{H} 15$ & 116.7 \\
\hline $\mathrm{C} 2-\mathrm{C} 3-\mathrm{H} 3$ & 119.9 & $\mathrm{~N} 3-\mathrm{C} 15-\mathrm{H} 15$ & 116.7 \\
\hline $\mathrm{C} 3-\mathrm{C} 4-\mathrm{C} 5$ & $120.3(3)$ & $\mathrm{N} 3-\mathrm{C} 16-\mathrm{C} 17$ & $122.1(3)$ \\
\hline $\mathrm{C} 3-\mathrm{C} 4-\mathrm{H} 4$ & 119.9 & $\mathrm{~N} 3-\mathrm{C} 16-\mathrm{H} 16$ & 118.9 \\
\hline $\mathrm{C} 5-\mathrm{C} 4-\mathrm{H} 4$ & 119.9 & $\mathrm{C} 17-\mathrm{C} 16-\mathrm{H} 16$ & 118.9 \\
\hline $\mathrm{C} 4-\mathrm{C} 5-\mathrm{C} 6$ & $122.0(3)$ & $\mathrm{C} 16-\mathrm{C} 17-\mathrm{C} 18$ & $117.2(3)$ \\
\hline $\mathrm{C} 4-\mathrm{C} 5-\mathrm{Cl} 2$ & $117.6(2)$ & $\mathrm{C} 16-\mathrm{C} 17-\mathrm{H} 17$ & 121.4 \\
\hline $\mathrm{C} 6-\mathrm{C} 5-\mathrm{Cl} 2$ & $120.5(2)$ & $\mathrm{C} 18-\mathrm{C} 17-\mathrm{H} 17$ & 121.4 \\
\hline $\mathrm{N} 1-\mathrm{C} 6-\mathrm{C} 1$ & $121.9(2)$ & $\mathrm{N} 2-\mathrm{C} 18-\mathrm{C} 17$ & $121.4(3)$ \\
\hline $\mathrm{N} 1-\mathrm{C} 6-\mathrm{C} 5$ & $122.8(3)$ & $\mathrm{N} 2-\mathrm{C} 18-\mathrm{H} 18$ & 119.3 \\
\hline $\mathrm{C} 1-\mathrm{C} 6-\mathrm{C} 5$ & $115.2(2)$ & $\mathrm{C} 17-\mathrm{C} 18-\mathrm{H} 18$ & 119.3 \\
\hline $\mathrm{C} 8-\mathrm{C} 7-\mathrm{C} 12$ & $120.6(3)$ & $\mathrm{O} 2-\mathrm{Ag} 1-\mathrm{O} 1^{\mathrm{i}}$ & $148.04(10)$ \\
\hline $\mathrm{C} 8-\mathrm{C} 7-\mathrm{N} 1$ & $121.4(3)$ & $\mathrm{O} 2-\mathrm{Ag} 1-\mathrm{N} 2$ & $99.71(8)$ \\
\hline $\mathrm{C} 12-\mathrm{C} 7-\mathrm{N} 1$ & $118.1(2)$ & $\mathrm{O} 1-\mathrm{Ag} 1-\mathrm{N} 2$ & $89.58(8)$ \\
\hline $\mathrm{C} 7-\mathrm{C} 8-\mathrm{C} 9$ & $120.5(3)$ & $\mathrm{O} 2-\mathrm{Ag} 1-\mathrm{N} 3^{\mathrm{ii}}$ & $97.48(9)$ \\
\hline $\mathrm{C} 7-\mathrm{C} 8-\mathrm{H} 8$ & 119.8 & $\mathrm{O} 1^{\mathrm{i}}-\mathrm{Ag} 1-\mathrm{N} 3^{\mathrm{ii}}$ & $108.69(9)$ \\
\hline $\mathrm{C} 9-\mathrm{C} 8-\mathrm{H} 8$ & 119.8 & $\mathrm{~N} 2-\mathrm{Ag} 1-\mathrm{N} 3^{\mathrm{ii}}$ & $107.93(9)$ \\
\hline $\mathrm{C} 10-\mathrm{C} 9-\mathrm{C} 8$ & $119.7(3)$ & $\mathrm{O} 2-\mathrm{Ag} 1-\mathrm{Ag} 1^{\mathrm{i}}$ & $81.70(6)$ \\
\hline $\mathrm{C} 10-\mathrm{C} 9-\mathrm{H} 9$ & 120.2 & $\mathrm{O} 1^{\mathrm{i}}-\mathrm{Ag} 1-\mathrm{Ag} 1^{\mathrm{i}}$ & $76.19(6)$ \\
\hline $\mathrm{C} 8-\mathrm{C} 9-\mathrm{H} 9$ & 120.2 & $\mathrm{~N} 2-\mathrm{Ag} 1-\mathrm{Ag} 1^{\mathrm{i}}$ & $151.80(6)$ \\
\hline $\mathrm{C} 9-\mathrm{C} 10-\mathrm{C} 11$ & $119.4(3)$ & $\mathrm{N} 3^{\mathrm{ii}}-\mathrm{Ag} 1-\mathrm{Ag} 1^{\mathrm{i}}$ & $99.73(6)$ \\
\hline $\mathrm{C} 9-\mathrm{C} 10-\mathrm{H} 10$ & 120.3 & $\mathrm{C} 6-\mathrm{N} 1-\mathrm{C} 7$ & $123.3(2)$ \\
\hline $\mathrm{C} 11-\mathrm{C} 10-\mathrm{H} 10$ & 120.3 & $\mathrm{C} 6-\mathrm{N} 1-\mathrm{H} 1$ & 118.4 \\
\hline $\mathrm{C} 10-\mathrm{C} 11-\mathrm{C} 12$ & $122.2(3)$ & $\mathrm{C} 7-\mathrm{N} 1-\mathrm{H} 1$ & 118.4 \\
\hline $\mathrm{C} 10-\mathrm{C} 11-\mathrm{H} 11$ & 118.9 & $\mathrm{C} 15-\mathrm{N} 2-\mathrm{C} 18$ & $116.7(3)$ \\
\hline $\mathrm{C} 12-\mathrm{C} 11-\mathrm{H} 11$ & 118.9 & $\mathrm{C} 15-\mathrm{N} 2-\mathrm{Ag} 1$ & $122.4(2)$ \\
\hline $\mathrm{C} 11-\mathrm{C} 12-\mathrm{C} 7$ & $117.6(3)$ & $\mathrm{C} 18-\mathrm{N} 2-\mathrm{Ag} 1$ & $120.8(2)$ \\
\hline $\mathrm{C} 11-\mathrm{C} 12-\mathrm{C} 13$ & $120.6(3)$ & $\mathrm{C} 15-\mathrm{N} 3-\mathrm{C} 16$ & $115.9(3)$ \\
\hline $\mathrm{C} 7-\mathrm{C} 12-\mathrm{C} 13$ & $121.9(2)$ & $\mathrm{C} 15-\mathrm{N} 3-\mathrm{Ag} 1^{\mathrm{iii}}$ & $116.2(2)$ \\
\hline $\mathrm{C} 12-\mathrm{C} 13-\mathrm{C} 14$ & $114.9(2)$ & $\mathrm{C} 16-\mathrm{N} 3-\mathrm{Ag} 1^{\mathrm{iii}}$ & $127.6(2)$ \\
\hline $\mathrm{C} 12-\mathrm{C} 13-\mathrm{H} 13 \mathrm{~A}$ & 108.5 & $\mathrm{C} 14-\mathrm{O} 1-\mathrm{Ag}^{\mathrm{i}}$ & $125.2(2)$ \\
\hline
\end{tabular}




\begin{tabular}{|c|c|c|c|}
\hline $\mathrm{C} 14-\mathrm{C} 13-\mathrm{H} 13 \mathrm{~A}$ & 108.5 & $\mathrm{C} 14-\mathrm{O} 2-\mathrm{Ag} 1$ & $117.96(17)$ \\
\hline $\mathrm{C} 6-\mathrm{C} 1-\mathrm{C} 2-\mathrm{C} 3$ & $-0.1(5)$ & $\mathrm{N} 1-\mathrm{C} 7-\mathrm{C} 12-\mathrm{C} 13$ & $2.6(4)$ \\
\hline $\mathrm{C} 11-\mathrm{C} 1-\mathrm{C} 2-\mathrm{C} 3$ & $-180.0(3)$ & $\mathrm{C} 11-\mathrm{C} 12-\mathrm{C} 13-\mathrm{C} 14$ & $-105.3(3)$ \\
\hline $\mathrm{C} 1-\mathrm{C} 2-\mathrm{C} 3-\mathrm{C} 4$ & $-2.0(5)$ & $\mathrm{C} 7-\mathrm{C} 12-\mathrm{C} 13-\mathrm{C} 14$ & $75.6(3)$ \\
\hline $\mathrm{C} 2-\mathrm{C} 3-\mathrm{C} 4-\mathrm{C} 5$ & $0.7(5)$ & $\mathrm{C} 12-\mathrm{C} 13-\mathrm{C} 14-\mathrm{O} 2$ & $-31.7(4)$ \\
\hline $\mathrm{C} 3-\mathrm{C} 4-\mathrm{C} 5-\mathrm{C} 6$ & $2.7(5)$ & $\mathrm{C} 12-\mathrm{C} 13-\mathrm{C} 14-\mathrm{O} 1$ & $149.1(3)$ \\
\hline $\mathrm{C} 3-\mathrm{C} 4-\mathrm{C} 5-\mathrm{Cl} 2$ & $-175.9(3)$ & $\mathrm{N} 3-\mathrm{C} 16-\mathrm{C} 17-\mathrm{C} 18$ & $-0.5(4)$ \\
\hline $\mathrm{C} 2-\mathrm{C} 1-\mathrm{C} 6-\mathrm{N} 1$ & $179.0(3)$ & $\mathrm{C} 16-\mathrm{C} 17-\mathrm{C} 18-\mathrm{N} 2$ & $0.5(4)$ \\
\hline $\mathrm{C} 11-\mathrm{C} 1-\mathrm{C} 6-\mathrm{N} 1$ & $-1.2(4)$ & $\mathrm{C} 1-\mathrm{C} 6-\mathrm{N} 1-\mathrm{C} 7$ & $133.5(3)$ \\
\hline $\mathrm{C} 2-\mathrm{C} 1-\mathrm{C} 6-\mathrm{C} 5$ & $3.2(4)$ & $\mathrm{C} 5-\mathrm{C} 6-\mathrm{N} 1-\mathrm{C} 7$ & $-51.1(4)$ \\
\hline $\mathrm{C} 11-\mathrm{C} 1-\mathrm{C} 6-\mathrm{C} 5$ & $-176.9(2)$ & $\mathrm{C} 8-\mathrm{C} 7-\mathrm{N} 1-\mathrm{C} 6$ & $-21.6(4)$ \\
\hline $\mathrm{C} 4-\mathrm{C} 5-\mathrm{C} 6-\mathrm{N} 1$ & $179.8(3)$ & $\mathrm{C} 12-\mathrm{C} 7-\mathrm{N} 1-\mathrm{C} 6$ & $157.8(3)$ \\
\hline $\mathrm{Cl} 2-\mathrm{C} 5-\mathrm{C} 6-\mathrm{N} 1$ & $-1.6(4)$ & $\mathrm{N} 3-\mathrm{C} 15-\mathrm{N} 2-\mathrm{C} 18$ & $-0.3(4)$ \\
\hline $\mathrm{C} 4-\mathrm{C} 5-\mathrm{C} 6-\mathrm{C} 1$ & $-4.5(4)$ & $\mathrm{N} 3-\mathrm{C} 15-\mathrm{N} 2-\mathrm{Ag} 1$ & $-176.8(2)$ \\
\hline $\mathrm{Cl} 2-\mathrm{C} 5-\mathrm{C} 6-\mathrm{C} 1$ & $174.0(2)$ & $\mathrm{C} 17-\mathrm{C} 18-\mathrm{N} 2-\mathrm{C} 15$ & $-0.1(4)$ \\
\hline $\mathrm{C} 12-\mathrm{C} 7-\mathrm{C} 8-\mathrm{C} 9$ & $-1.8(4)$ & $\mathrm{C} 17-\mathrm{C} 18-\mathrm{N} 2-\mathrm{Ag} 1$ & $176.4(2)$ \\
\hline $\mathrm{N} 1-\mathrm{C} 7-\mathrm{C} 8-\mathrm{C} 9$ & $177.6(3)$ & $\mathrm{N} 2-\mathrm{C} 15-\mathrm{N} 3-\mathrm{C} 16$ & $0.3(4)$ \\
\hline $\mathrm{C} 7-\mathrm{C} 8-\mathrm{C} 9-\mathrm{C} 10$ & $-0.2(4)$ & $\mathrm{N} 2-\mathrm{C} 15-\mathrm{N} 3-\mathrm{Ag} 1^{\mathrm{iii}}$ & $173.7(2)$ \\
\hline $\mathrm{C} 8-\mathrm{C} 9-\mathrm{C} 10-\mathrm{C} 11$ & $1.0(5)$ & $\mathrm{C} 17-\mathrm{C} 16-\mathrm{N} 3-\mathrm{C} 15$ & $0.2(4)$ \\
\hline $\mathrm{C} 9-\mathrm{C} 10-\mathrm{C} 11-\mathrm{C} 12$ & $0.2(5)$ & $\mathrm{C} 17-\mathrm{C} 16-\mathrm{N} 3-\mathrm{Ag} 1^{\mathrm{iii}}$ & $-172.4(2)$ \\
\hline $\mathrm{C} 10-\mathrm{C} 11-\mathrm{C} 12-\mathrm{C} 7$ & $-2.1(4)$ & $\mathrm{O} 2-\mathrm{C} 14-\mathrm{O} 1-\mathrm{Ag}^{\mathrm{i}}$ & $17.1(4)$ \\
\hline $\mathrm{C} 10-\mathrm{C} 11-\mathrm{C} 12-\mathrm{C} 13$ & $178.7(3)$ & $\mathrm{C} 13-\mathrm{C} 14-\mathrm{O} 1-\mathrm{Ag}^{\mathrm{i}}$ & $-163.8(2)$ \\
\hline $\mathrm{C} 8-\mathrm{C} 7-\mathrm{C} 12-\mathrm{C} 11$ & $3.0(4)$ & $\mathrm{O} 1-\mathrm{C} 14-\mathrm{O} 2-\mathrm{Ag} 1$ & $18.3(4)$ \\
\hline $\mathrm{N} 1-\mathrm{C} 7-\mathrm{C} 12-\mathrm{C} 11$ & $-176.5(2)$ & $\mathrm{C} 13-\mathrm{C} 14-\mathrm{O} 2-\mathrm{Ag} 1$ & $-160.8(2)$ \\
\hline $\mathrm{C} 8-\mathrm{C} 7-\mathrm{C} 12-\mathrm{C} 13$ & $-177.9(2)$ & & \\
\hline
\end{tabular}

Symmetry codes: (i) $-x+1,-y+1,-z+2$; (ii) $x,-y+1 / 2, z+1 / 2$; (iii) $x,-y+1 / 2, z-1 / 2$.

Hydrogen-bond geometry $\left(A,{ }^{\circ}\right)$

$\mathrm{Cg} 6$ is the centroid of the [please define] ring.

\begin{tabular}{lllll}
\hline$D-\mathrm{H} \cdots A$ & $D-\mathrm{H}$ & $\mathrm{H} \cdots A$ & $D \cdots A$ & $D-\mathrm{H} \cdots A$ \\
\hline $\mathrm{N} 1-\mathrm{H} 1 \cdots \mathrm{O} 2$ & 0.86 & 2.43 & $2.971(3)$ & 122 \\
$\mathrm{C} 16-\mathrm{H} 16 \cdots \mathrm{O} 1^{\mathrm{iv}}$ & 0.93 & 2.51 & $3.248(4)$ & 136 \\
$\mathrm{C} 13-\mathrm{H} 13 B \cdots C g 6^{\mathrm{iii}}$ & 0.97 & 3.30 & $3.983(3)$ & 129 \\
\hline
\end{tabular}

Symmetry codes: (iii) $x,-y+1 / 2, z-1 / 2$; (iv) $x, y, z-1$. 